\title{
IUFOST2006/1353 \\ New developments of quality culture in the Middle East
}

\author{
A. Idriss \\ Middle East North Africa Food safety Associates, Lions Bldg \# 301, 126 Sourati Str., 00000 Beirut, Lebanon \\ info@mefosa.com
}

New Development of Quality Culture in the Middle East

Atef Wafic Idriss, MEFOSA CEO

Abstract:

The MENA Region has good export potential for processed food products and they are constrained by high trade barriers in many developed countries. For instance, from January to June 2001, 27\% of food exports from Egypt, Jordan, Lebanon and Syria to the United States were rejected by the USFDA due to non-compliance with the U.S. safety measures (filth, microbiological contamination, greater than permitted levels of pesticide residues or food additives) and $58 \%$ due to labeling problems, barriers may also include food borne diseases and surveillance weaknesses in most of the countries.

In the light of the above, the existence of Food Safety consultancy is no more a want but either a persistent need and MEFOSA sarl is leading with its Agri-Agro business related fields services: Food Safety, Food Hygiene, Food Traceability, as for the new EU directives and laws, Food Safety Audits and Training to national and international food related requirements: HACCP Implementations, GMP and Food borne Illness, Sanitation/GMP Audits: Sanitation and Handling practices. To conduct an independent audit using 21 CFR 110 as a guide, general EFSA/EU and FDA/USA Compliance Audits: To comply with FDA, USDA and EFSA regulations regarding acceptable equipment and Networking, Communication within companies (ERP solutions) and address supply chains sterilization practices, Agricultural primary production, technical advisory services on improving agricultural productivity, improved training methodologies, technical know how and Rural extension services

Finally, some of MEFOSA's projects: AGRIPOLE: Agro-Economic and Food Safety SME Incubator, Market Access E-commerce Site: Lebanese Food Portal and ADP: Agriculture Development Program. 